\title{
Desiccation resistance: effect of cuticular hydrocarbons and water content in Drosophila melanogaster adults
}

\author{
Jean-Francois Ferveur ${ }^{\text {Corresp., }}{ }^{1}$, Jérôme Cortot ${ }^{1}{ }^{\text {, }}$ Karen Rihani ${ }^{1}$, Matthew Cobb ${ }^{2}$, Claude Everaerts ${ }^{1}$ \\ ${ }^{1}$ Centre des Sciences du Goût et de l'Alimentation, Agrosup-UMR 6265 CNRS, UMR 1324 INRA, Université de Bourgogne, Dijon, France \\ 2 School of Biological Sciences, University of Manchester, Manchester, United Kingdom \\ Corresponding Author: Jean-Francois Ferveur \\ Email address: jean-francois.ferveur@u-bourgogne.fr
}

Background.The insect cuticle covers the whole body and all appendages and has bi-directionnal selective permeability: it protects against environmental stress and pathogen infection and also helps to reduce water loss. The adult cuticle is often associated with a superficial layer of fatty acid-derived molecules such as waxes and long chain hydrocarbons that prevent rapid dehydration. The waterproofing properties of cuticular hydrocarbons (CHs) depend on their chain length and desaturation number. Drosophila $\mathrm{CH}$ biosynthesis involves an enzymatic pathway including several elongase and desaturase enzymes.

Methods. The link between desiccation resistance and $\mathrm{CH}$ profile remains unclear, so we tested (1) experimentally selected desiccation-resistant lines, (2) transgenic flies with altered desaturase expression and (3) natural and laboratory-induced $\mathrm{CH}$ variants. We also explored the possible relationship between desiccation resistance, relative water content and fecundity in females.

Results. We found that increased desiccation resistance is linked with the increased proportion of desaturated $\mathrm{CHs}$, but not with their total amount. Experimentally-induced desiccation resistance and $\mathrm{CH}$ variation both remained stable after many generations without selection. Conversely, flies with a higher water content and a lower proportion of desaturated $\mathrm{CH}$ s showed reduced desiccation resistance. This was also the case in flies with defective desaturase expression in the fat body.

Discussion. We conclude that rapidly acquired desiccation resistance, depending on both $\mathrm{CH}$ profile and water content, can remain stable without selection in a humid environment. These three phenotypes, which might be expected to show a simple relationship, turn out to have complex physiological and genetic links. 

content in Drosophila melanogaster adults

10 Jean-François Ferveur ${ }^{1 *}$, Jérôme Cortot ${ }^{1}$, Karen Rihani ${ }^{1}$, Matthew Cobb ${ }^{2}$ and Claude 11 Everaerts ${ }^{1}$

171 Centre des Sciences du Goût et de l'Alimentation, Agrosup-UMR 6265 CNRS, UMR 1324

18 INRA, Université de Bourgogne, 6, Bd Gabriel, F-21000 Dijon.

19

$20{ }^{2}$ School of Biological Sciences, University of Manchester, Manchester M13 9PT, U.K.

$22 *$ corresponding author : Jean-François Ferveur / jean-francois.ferveur@u-bourgogne.fr 


\section{Abstract}

26 Background. The insect cuticle covers the whole body and all appendages and has bi27 directionnal selective permeability: it protects against environmental stress and pathogen 28 infection and also helps to reduce water loss. The adult cuticle is often associated with a 29 superficial layer of fatty acid-derived molecules such as waxes and long chain hydrocarbons that 30 prevent rapid dehydration. The waterproofing properties of cuticular hydrocarbons $(\mathrm{CHs})$ depend 31 on their chain length and desaturation number. Drosophila $\mathrm{CH}$ biosynthesis involves an 32 enzymatic pathway including several elongase and desaturase enzymes.

33 Methods. The link between desiccation resistance and $\mathrm{CH}$ profile remains unclear, so we tested 34 (1) experimentally selected desiccation-resistant lines, (2) transgenic flies with altered desaturase expression and (3) natural and laboratory-induced $\mathrm{CH}$ variants. We also explored the possible relationship between desiccation resistance, relative water content and fecundity in females.

37 Results. We found that increased desiccation resistance is linked with the increased proportion 38 of desaturated $\mathrm{CHs}$, but not with their total amount. Experimentally-induced desiccation resistance and $\mathrm{CH}$ variation both remained stable after many generations without selection.

40 Conversely, flies with a higher water content and a lower proportion of desaturated CHs showed 41 reduced desiccation resistance. This was also the case in flies with defective desaturase expression in the fat body.

Discussion. We conclude that rapidly acquired desiccation resistance, depending on both $\mathrm{CH}$ profile and water content, can remain stable without selection in a humid environment. These three phenotypes, which might be expected to show a simple relationship, turn out to have complex physiological and genetic links. 


\section{Introduction}

The resistant yet flexible outer layers of the insect exoskeleton, generally known as the cuticle, are involved in many vital functions and possess great structural, mechanical, and chemical complexity (Locke 1966). The cuticle constrains the animal's shape, serves as an attachment point for muscles, and is the basis for a variety of specialized organs such as sensory hairs, respiratory trachea, feeding and copulatory structures (Demerec 1950). It also provides protection against physical and chemical environmental stressors such as desiccation. The cuticle not only limits desiccation (Wigglesworth 1945), it also permits selective exchanges between the organism and the outer world (Moussian 2010).

The soft and unpigmented cuticle of young adult insects undergoes a rapid process of melanization (Andersen 2010) during which stabilized lipids, produced by the oenocytes and fat body, combine with proteins and stiffen the cuticle before sclerotization, which constitutes the final developmental phase (Wigglesworth 1988). Most of our knowledge of the biochemical and genetic parthways involved in these processes comes from Drosophila, where they depend on the activity of the neurohormone, bursicon (Honegger et al. 2008), coupled with its rickets receptor ( $r k$; (Harwood et al. 2013), as well as on lipid production. A large part of the lipids contained within or secreted onto the epicuticle are long-chain hydrocarbons, generally known as cuticular hydrocarbons (CHs), the production of which depends on the Cyp4g1 gene (Qiu et al. 2012), and on several genes coding for an elongase (Chertemps et al. 2007; Howard \& Blomquist 2005) and for substrate-specific lipid desaturases (desat1, desat2; Bousquet et al. 2012; Dallerac et al. 2000; Flaven-Pouchon et al. 2016). In Anopheles gambiae, a Cyp4g1 ortholog, associated with insecticide resistance, catalyzes $\mathrm{CH}$ production (Balabanidou et al. 2016).

The waxes, lipids and $\mathrm{CHs}$ found on the epicuticle appear to have several functions. They act as a barrier against pathogenic microorganisms and insecticides (Balabanidou et al. 2016; da Silva et al. 2015; Gołębiowski et al. 2008), they reduce the heat load by reflecting solar radiation and they deter predators (Eigenbrode \& Espelie 2003; Hadley 1994). They also help to limit transpiration (Gibbs 1998; Hadley 1994). Although the adult $\mathrm{CH}$ profile shows seasonal and environmental variation (Gibbs \& Pomonis 1995; Howard \& Blomquist 2005; Savarit \& Ferveur 2002; Toolson \& Hadley 1979) some of its components serve as sex- and/or speciesspecific pheromones (Ferveur 2005; Jallon 1984; Savarit et al. 1999). The desat1 gene affects 
78 both the production and the reception of Drosophila melanogaster sex pheromones, while it has 79 been suggested that desat2 is involved in speciation (Fang et al. 2002). desat1 is also expressed in tissues involved in water balance, in particular the Malpighian tubules (Bousquet \& Ferveur 2012; Bousquet et al. 2012; Dow 2009).

Several studies of D. melanogaster have used artificial selection to enhance desiccation resistance by altering water loss, osmotic regulation and $\mathrm{CH}$ content. There is a clear link between desiccation resistance and water retention, dry mass and ionic content (Folk \& Bradley 2004; Gibbs et al. 1997), but the role of $\mathrm{CH}$ profile in preventing water loss in such selection experiments remains unclear (Gibbs et al. 1997; Gibbs \& Rajpurohit 2010). Selected lines showed no differences in the quantity of $\mathrm{CHs}$, but sex differences in $\mathrm{CH}$ chain length were linked to variation in resistance - faced with a desiccation challenge, females survived longer than males (Gibbs et al. 1997; Qiu et al. 2012).

To reveal the link between desiccation resistance and $\mathrm{CH}$ profile in D.melanogaster flies, we carried out a three-part investigation using $(i)$ experimentally selected lines, (ii) desat 1 transgenics and (iii) a desat2 natural variant and $r k$ mutants.

\section{Materials and Methods}

\section{Flies}

D. melanogaster were raised on yeast / cornmeal / agar medium and kept at $24 \pm 0.5^{\circ}$ with $65 \pm 5 \%$ humidity on a $12 \mathrm{~L}: 12 \mathrm{D}$ cycle (subjective day=8:00am to $8: 00 \mathrm{pm}$ ). Flies were isolated under light $\mathrm{CO}_{2}$ anaesthesia either $0-4 \mathrm{~h}$ (for virgin females) or less than $24 \mathrm{~h}$ (for all other flies) after eclosion. Male and females were held separately in fresh glass food vials in groups of 10 flies until the day of the experiment (4-5 days old, unless specified). Same-sex flies were then transferred to an empty glass vial to obtain groups of $50 \pm 5$ individuals.

We tested two wild-type stocks, Dijon2000 (Di2) and Zimbabwe30 (Z30), which were collected in France and Zimbabwe respectively (Grillet et al. 2012; Houot et al. 2010). We also used the Di2/ $w^{1118}$ line (Di2/w), derived from the Di2 strain and introgressed into the genome of the $w^{1118}$ strain over five repeated backcross generations. 
We used several desat1-Gal4 transgenic drivers built with putative desat1 regulatory

106

107

108

109

110

111

112

113

114

115

116

117

118

119

120

121

122

123

124

125

126

127

128

129

130

131

132

133

134 regions (PRRs) fused with Gal4. PRR(RA)-Gal4 is expressed in the wing margin and in the brain as well as in other tissues; PRR(RC)-Gal4 is exclusively expressed in the fat body; PRR(RE)Gal4 is expressed in the oenocytes; PRR(RB) is expressed in the Malpighi tubules and midgut; PRR(RD) is expressed in a vaginal moon-shaped structure in the female and in the male ejaculatory bulb; PRR(RDiO)-Gal4 is expressed in neural tissues involved in the discrimination of sex pheromones by male flies (Bousquet et al. 2012). We also used a Gal4 transgene containing the complete desat1 sequence fused to GAL4 (6908bp; 6908-Gal4; (Bousquet et al. 2016)). Gal4 drivers were used to target the UAS-desat1-RNAi transgene (IR; VDRC \#33338; This UAS-RNAi line was chosen from three alternative lines for its clear effect on both the hydrocarbon and behavioral phenotypes tested here; Bousquet et al. 2012; Houot et al. 2017); this allowed us to down-regulate desat1 expression in the tissues where Gal4 is expressed ([IR $\mathrm{x}$ driver-Gal4]; (Houot et al. 2017)). We also controlled for the effect of each driver-Gal4 in the Di2 background ([Di2 x driver-Gal4]). To homogenize genetic backgrounds, all UAS and Gal4 transgenes were isogenized in the genetic background of the Di2/w $w^{1118}$ strain prior to testing.

Two rickets alleles were used, $r k[1]$ and $r k[4]$, which contain different mutations that create premature stop codons, and are considered null alleles (Baker \& Truman 2002). We carried out reciprocal crosses between virgin adults carrying the $r k$ [1] or the $r k$ [4] mutation, both of which were maintained in a heterozygous state by the [SM2, CyO] balancer chromosome. From this cross, we obtained $r k[1] / r k[4]$ heteroallelic mutant adults and $r k[1] / \mathrm{CyO}$ and $r k[4] / \mathrm{CyO}$ control flies. To control the effect of the SM2, CyO balancer chromosome, we mated Di2/w females with either $r k[1] / \mathrm{CyO}$ or $r k[4] / \mathrm{CyO}$ males and collected all CyO flies (i.e. those not carrying the $r k$ mutation; $\mathrm{CyO} / \mathrm{w})$.

\section{Experimental selection}

Groups of same-sex flies $(n=50 \pm 5)$ of a given age were kept in glass vials (Fig 1A). Six or seven vials were kept in a transparent plastic box, which was partly filled with silica gel to reduce the humidity to $20 \% \mathrm{RH}$ and was secured with transparent adhesive tape. We simultaneously tested three or four boxes placed on a hot plate at $25.0 \pm 0.2^{\circ}$ (StörkTronic, Präzitherm, Düsseldorf, Germany). Every hour or two hours, we counted the number of dead flies in each vial. In each box and in each experiment, we mixed vials containing the different 
135 genotypes. For each generation, we performed two or three series of tests that were subsequently 136 pooled.

To experimentally select lines for desiccation resistance, the $1-2 \%$ flies showing the

138 longest life span during the desiccation challenge were placed in a fresh food vial and allowed to 139 mate, producing the next generation (Fig 1B). This procedure, begun with the wild-type Di2 140 strain, was carried out over six successive generations (F1-F6) and was then sporadically carried 141 out until F57. Of ten F1 lines, flies of the \#7 line, which showed high desiccation resistance, 142 were used to create six F2 lines called 77S, which were subject to subsequent selection. Between 143 F2 and F6, we also tested flies resulting from the backcross between 77S females and Di2 males 144 (77S x Di2) and the reciprocal backcross. After F6, no selection was carried out on 77S lines 145 except to create six 77S-Sel lines which were subject to selection; each of these derived from their respective $77 \mathrm{~S}$ lines (e.g. $77 \mathrm{~S}-\mathrm{Sel} 1$ derived from the $77 \mathrm{~S}_{1}$ line).

\section{Cuticular hydrocarbons}

5-day-old flies were frozen for $5 \mathrm{~min}$ at $-20^{\circ} \mathrm{C}$ and their cuticular hydrocarbons then individually extracted for $5 \mathrm{~min}$ at room temperature using $30 \mu \mathrm{l}$ of a mixture of hexane and methylene chloride (50/50 by volume). The solution also contained $3.33 \mathrm{ng} / \mu \mathrm{l}$ of C26 ( $n$ hexacosane) and $3.33 \mathrm{ng} / \mu 1$ of C30 (n-triacontane) as internal standards. Cuticular hydrocarbons were quantified by gas chromatography using a Varian CP3380 gas chromatograph fitted with a flame ionization detector, a CP Sil 5CB column $(25 \mathrm{~m} \mathrm{x} 0.25 \mathrm{~mm}$ internal diameter; $0.1 \mu \mathrm{m}$ film

154 thickness; Agilent), and a split-splitless injector $(60 \mathrm{ml} / \mathrm{min}$ split-flow; valve opening $30 \mathrm{sec}$ 155 after injection) with helium as carrier gas $\left(50 \mathrm{~cm} / \mathrm{sec}\right.$ at $\left.120^{\circ} \mathrm{C}\right)$. The temperature program began at $120^{\circ} \mathrm{C}$, ramping at $10^{\circ} \mathrm{C} / \mathrm{min}$ to $140^{\circ} \mathrm{C}$, then ramping at $2^{\circ} \mathrm{C} / \mathrm{min}$ to $290^{\circ} \mathrm{C}$, and holding for 10

157 min. Individual $\mathrm{CH}$ profiles were determined by integration of 46 peak areas in males and 158 females. This corresponded to all the peaks that could be consistently identified in all individuals 159 (Everaerts et al. 2010). The chemical identity of the peaks was checked using gas 160 chromatography - mass spectrometry equipped with a CP Sil 5CB column. The amount 161 (ng/insect) of each component was calculated on the basis of the data obtained from the internal 162 standards. We calculated the absolute amount of each group of CHs (alkene Q, alkane Q), the 163 relative amount of each $\mathrm{CH}$ group (alkene \%, alkane \%) from the overall $\mathrm{CH}$ total $\left(\sum \mathrm{CH}\right)$ and 164 their ratio (Desaturated:Linear $=\mathrm{D}: \mathrm{L})$. At least 10 flies were tested per condition. 


\section{Water content}

166 Groups of 10 live anaesthetized females were weighed on a precision balance $( \pm 10 \mu \mathrm{g}$;

167 Sartorius R160-P) to obtain their fresh weight. Each group of females was then kept for 24 hours

168 in an empty glass vial in a $37^{\circ}$ dry incubator to allow complete desiccation. The dead, dry flies

169 were then weighed to obtain the dry weight. The relative level of water in each group was 170 estimated based on the fresh weight : dry weight ratio. 


\section{Fecundity}

172

Females and males were kept in groups of ten pairs until they were 4 days old. Females

173 were then isolated (males were discarded) and the total number of male and female adult

174 progeny was noted for 7 days following the emergence of the first offspring. The sex ratio of the

175 progeny was also noted.

\section{Statistics}

All statistical analyses were performed using XLSTAT 2012 (Addinsoft 2012). For each desiccation replicate, logistic regression was used to characterize the relationship between mortality and time by estimating the lethal time 50 (LT50) and the regression slope (Robertson \& Preisler 1992). Thereafter, for each generation an inter-line comparison for these two parameters was carried out either with a Kruskall-Wallis test with Conover-Iman multiple pairwise comparisons ( $p=0.05$, with a Bonferroni correction) or with a Mann-Whitney test, after excluding extreme outliers using Tukey's method (Tukey 1977). The overall amount of CH $\left(\sum \mathrm{CH}\right)$, the Desaturated:Linear ratio (D:L), the fresh weight : dry weight ratio, the total number of adult progeny and the sex ratio were also compared using the same statistical tests.

\section{Results}

\section{Selection for desiccation resistance}

5-7 day old flies were placed in groups in a relatively dry environment $(20 \% \mathrm{RH})$ at $25 \pm 0.2^{\circ} \mathrm{C}$, and two measures of survivorship were taken: LT50 (time at which $50 \%$ flies were dead) and lethality slope (the steepness of this curve indicates the proportion of flies dying per hour; Fig 1A). Males were significantly more affected by the dry conditions than females, as shown by a shorter LT50 and a steeper slope $\left(\mathrm{KW}_{(5 \mathrm{df})}=31.74, p<10^{-4}\right.$; Fig S1). Because of this sex difference, we subsequently focused on female resistance. No significant differences were found between virgin and mated females (see Materials and Methods). Survivors of this initial desiccation challenge were allowed to mate, and a selection experiment on desiccation resistance was then undertaken, with eight replicate lines. After only one generation, significant resistance appeared in one line (\#7; Fig 2); we therefore focused on this line, crossing \#7 flies to create six replicate selected lines, known as $77 \mathrm{~S}_{0-5}$. These lines were then used in our experiment on 
199 desiccation resistance; data from all six 77S lines were pooled at each generation for statistical 200 analysis.

From F3 to F6, 77S females showed significantly increased desiccation resistance as 202 compared to control Di2 flies, as shown by a higher LT50 $\left(p<10^{-4}-10^{-3}\right)$ and a reduced slope ( $p$ : 203 0.001-0.037). In order to explore the genetic control of these characters, female and male 77S 204 flies were separately backcrossed to control Di2 flies, and their desiccation resistance was 205 measured. Although both the LT50 and slope of the offspring of the [77S $\mathrm{f} \times \mathrm{Di} 2 \mathrm{~m}$ ] backcross were intermediate between control and 77S lines, flies produced by the reciprocal backcross [Di2 f $x$ 77S m] were not significantly different from control $(\mathrm{p}=\mathrm{ns})$. The effect of selection on male flies was much less, if any (Fig S2). These data suggest that the character(s) that have been selected for in the 77S flies are primarily transmitted through female flies.

210

After F6, systematic selection of the 77S lines was relaxed (Fig 1B). Over 52

211 subsequent generations, desiccation resistance was sporadically tested in the $77 \mathrm{~S}_{0-5}$ lines; we also

212 re-selected females from each $77 \mathrm{~S}$ line for one or two generations prior to these desiccation

213 resistance tests and tested their progeny (77S-Sel; Fig 3). Compared to control females, 77S

214 females showed a significantly increased LT50 (median value ranges: 14.83-21.67 h and 18.77-

$21524.45 \mathrm{~h}$, respectively) and/or a shallower slope (median value ranges: 9-16 and 5-9\%

216 lethalithy/h, respectively) that showed little consistent variation over time. Reintroduction of 217 selection in the 77S-Sel lines had no effect - these flies were not significantly different from 77S

218 flies that were reared under relaxed selection, indicating that the character(s) isolated in the 219 selection procedure are at fixation in these lines.

220

221

222

223

224

225

226

227

228

\section{Effects on associated characters}

Cuticular hydrocarbons $(\mathrm{CH})$ have regularly been implicated in the evolution of desiccation resistance; we therefore measured $\mathrm{CH}$ profiles in $\mathrm{F} 7-\mathrm{F} 9 \mathrm{77}_{1-5}$ females and in $\mathrm{F} 8$ males, and sporadically thereafter between F18 and F59. Beside the absolute $\mathrm{CH}$ amount $(\mathrm{CH})$, we also determined the absolute (Q) and relative amounts (\%) of desaturated $\mathrm{CHs}$ (alkenes) and of linear saturated CHs (alkanes) and their ratio (Desaturated:Linear $=\mathrm{D}: \mathrm{L}$ ). Although we observed both interline and intergenerational differences in the $77 \mathrm{~S}_{0-5}$ flies (Fig 4), all F9 females showed increased levels of alkene $\mathrm{Q}$ and most showed an increased alkene $\%\left(77 \mathrm{~S}_{4}\right.$ was an exception). Compared to control Di2 females, most lines showed higher $\sum$ CH and D:L (Fig S3 - 
$22977 \mathrm{~S}_{0}$ was an exception). At F18, following 12 generations of relaxed selection, only $77 \mathrm{~S}_{1}$ and $23077 \mathrm{~S}_{2}$ females showed increased D:L, while at F19 only $77 \mathrm{~S}_{2}$ females showed increased D:L (Fig $2315)$.

At F55, we tested $77 \mathrm{~S}_{1}-77 \mathrm{~S}_{5}$ flies (the $77 \mathrm{~S}_{0}$ line was lost between $\mathrm{F} 35$ and F55), all of

233

234

235

236

237

238

239

240

241

242

243

244

245

246

247

248

249

250

251

252

253

254

255

256

257 which showed a significantly increased D:L, due to their higher alkene levels (Q and \%) and lower alkene Q (Figs $5 \& \mathrm{~S} 4$ ). In all $77 \mathrm{~S}$ lines, $\Sigma \mathrm{CH}$ was significantly higher than in Di2 females. At F57, 77S females were compared with females that had been re-selected at F55; 77SSel - Fig 5). D:L increased in three $77 \mathrm{~S}$ lines $\left(77 \mathrm{~S}_{1}-77 \mathrm{~S}_{3}\right)$, but not in $77 \mathrm{~S}-\mathrm{Sel}$ lines. All $77 \mathrm{~S}$ lines showed increased $\Sigma \mathrm{CH}$, while only one of the 77S-Sel lines showed such an effect. These differences between 77S and 77S-Sel lines contrast with the results of the desiccation resistance experiments, where there were no significant differences between these sets of lines, and suggest that $\mathrm{CH}$ composition and desiccation resistance are not identical.

In order to explore the link between $\mathrm{CH}$ composition and desiccation resistance, we measured the fresh and dry weight of flies (either freshly killed, or desiccated, respectively) from these lines at F19 and F57/59. Fresh and dry weight can be considered as indirect measures of cuticular surface and their ratio reflects the water retention ability of a particular strain. In F19 females, the Fresh : Dry weight ratio was significantly higher in lines $77 \mathrm{~S}_{1}-77 \mathrm{~S}_{3}$ than in Di2 flies (Fig 6A). However in F57/F59 females, no difference was detected between Di2 and 77S females (Fig 6B).

Finally, to confirm that we had not inadvertently selected for changes in sex ratio or number of eggs laid by the $77 \mathrm{~S}$ females, we counted the total number of adult progeny left by single 77S, 77S-Sel and control females, and calculated their sex-ratio (female : male). There were no overall differences between these groups (Fig S5). Only $77 \mathrm{~S}_{3}$ females showed a significant variation by producing more progeny than Di2 females (116 and 66, respectively) and more males (64 and 32, respectively), but this did not affect the sex ratio.

\section{Genetic control - desat1}

A major gene involved in $\mathrm{CH}$ synthesis is desat1, which controls a vital desaturation step in the hydrocarbon biosynthetic pathway. To explore the role of desat1 in desiccation resistance, we downregulated this gene in subsets of tissues and measured the consequences for 
258 desiccation resistance and associated characters. Driver-Gal4 lines, made either with each 259 putative desat1 regulatory region (PRR-Gal4) or the complete desat1 regulatory region (6908260 Gal4), were used to drive the expression of the UAS-desat1 RNAi transgenic reporter line (IR).

261 This allowed us to downregulate desat1 expression either in Gal4-targeted tissues ([PRR-Gal4 x 262 IR]) or in all desat1-expressing tissues ([6908-Gal4 x IR]). Under the desiccation conditions used 263 in the selection experiment, [RC-Gal4 x IR] and [6908-Gal4 x IR] females showed reduced 264 desiccation resistance, as shown by a reduced LT50 (Fig 7A), but no difference in the rate at 265 which flies died (as measured by the slope; Fig 7B). This suggests that expression of desat1, in 266 particular in the fat body, is required for normal desiccation resistance. Control genotypes were 267 tested simultaneously and showed no effects (Figs 7C, 7D).

268 To confirm that manipulation of desat 1 had altered the $\mathrm{CH}$ profiles, the $\mathrm{CH}$ levels of [IR $269 \mathrm{x}$ driver-Gal4] females in two generations $\left(\mathrm{F}_{\mathrm{A}}\right.$ and $\left.\mathrm{F}_{\mathrm{B}}\right)$ were compared to simultaneously-raised 270 controls (Di2; Di2/w; [Di2 x IR]; [driver-Gal4 x Di2]; Fig 8). Despite slight quantitative 271 variations between the two generations Figs 8A, 8B), $\mathrm{F}_{\mathrm{A}}$ and $\mathrm{F}_{\mathrm{B}}$ flies showed similar effects. In 272 particular, [RE-Gal4 x IR], [6908-Gal4 x IR] and to a lesser extent [RC-Gal4 x IR] flies 273 produced lower alkene levels (Q and \%; Fig S6). [RE-Gal4 x IR] and [6908-Gal4 x IR] flies also 274 showed increased alkane levels (Q and \%) and lower D:L compared to controls. Comparison of 275 Fresh : Dry weight revealed a significant increase in [RC-Gal4 x IR] females compared to 276 control lines (Fig 9), paralleling the effects on $\mathrm{CH}$ levels shown by this line. Although [6908-

277 Gal4 x IR] flies also appeared to show an increased ratio, this was not significant compared to 278 [Di2 x IR] controls. No differences in average fecundity were found when $\mathrm{F}_{\mathrm{A}}$ and $\mathrm{F}_{\mathrm{B}}$ flies were 279 compared with controls. (Fig S7).

\section{Genetic control - natural and lab-induced variants}

To further explore genetic control of the link between desiccation resistance and $\mathrm{CH}$ 282 profile, we studied naturally-occurring and laboratory-induced variants. In Zimbabwe (Z30) 283 flies, the female-specific alkene isomer 5,9-heptacosadiene (5,9HD) largely replaces 7,11HD 284 which is abundant in the other strains studied here (Flaven-Pouchon et al. 2016; Grillet et al. 285 2012). The desiccation resistance shown by these females was not significantly different from 286 Di2 flies. $r k^{1 / 4}$ mutant females produce high absolute amounts of $\mathrm{CH}\left(\sum \mathrm{CH}\right.$, alkene and alkane) 287 but control-like alkene and alkane \% and D:L; these mutants showed significantly lower 
288 resistance than control females (lower LT50, but no slope variation) (Fig 10). However, $r k / \mathrm{CyO}$

289 females showed significantly greater resistance than controls, with a highly increased LT50 and a 290 strongly decreased slope. Increased resistance in $r k / \mathrm{CyO}$ females was not related to the presence 291 of the $\mathrm{CyO}$ balancer $-\mathrm{CyO} / \mathrm{w}$ females did not show the same effect. Finally, there was a slightly 292 increased resistance (LT50 and slope) shown by Di2/w females compared to Di2 females.

\section{Discussion}

294

295

296

297

298

299

300

301

302

303

304

305

306

307

308

309

310

311

312

313

314

315

316

Desiccation is a major physiological challenge faced by terrestrial arthropods, and both experimental and theoretical arguments have been deployed to suggest that there is a physiological and evolutionary link between desiccation resistance and the presence of certain cuticular hydrocarbons, which act as a waxy outer layer restricting permeability (Gibbs 1998; Qiu et al. 2012; Toolson \& Hadley 1979). Using a range of experimentation approaches, we were able to reveal genetic variation for desiccation resistance in our laboratory strain of $D$. melanogaster, as shown by the appearance of a desiccation-resistant strain (\#7) after one generation, and then a rapid response to selection for desiccation resistance in multiple replicates of that line which was maintained over dozens of generations despite the subsequent relaxation of selection. Furthermore, we found correlated changes in cuticular hydrocarbon composition in these replicate lines, in particular the overall amount of $\mathrm{CH}$ and the $\mathrm{D}: \mathrm{L}$ ratio, together with water retention ability. We suggest that these were causally linked - altered cuticular hydrocarbon composition enabled the selected lines to retain water and resist desiccation. These findings suggest that genetic variability for desiccation resistance exists even in laboratory lines (the Di2 line was captured in 2000); variability in wild populations is presumably greater, and may help explain the global success of this species.

The detection of significant desiccation resistance in line \#7 after a single generation enabled us to select for that character over subsequent generations, significantly altering both LT50 and the lethality slope. From the outset, we found that females were significantly more responsive to selection than males, which remained significantly more affected by the desiccation challenge than females. This led us to focus on the female phenotype for the rest of the experiment. Two possible explanations for this sex difference are that the genes we were selecting were sex-limited, expressed only in females, linked to qualitative and quantitative sex 
317 differences in CHs (see below), or that the effect was simply due to size - female Drosophila are

318 larger than males, with a lower surface area : volume ratio, which in turn would reduce the effect 319 of desiccation.

Although selection was relaxed after F6, the resistance character we had selected in the

321 \#7 line was stable for over 50 generations, with no significant effect of the reintroduction of 322 selection, indicating that the character(s) involved were at fixation. The fact that these characters 323 were detected after one generation and became fixed after a further five generations of selection 324 suggests that our selection protocol and the available genetic variation in the parent population 325 were well matched and the strongest possible phenotypes were rapidly selected through the 326 available genetic variation.

At first glance it could be thought that the main effects we observed - increased desiccation resistance, increased quantities of $\mathrm{CH}$, and increased water content - were all produced by an increase in the size of female flies, which would also increase the quantity of $\mathrm{CH}$ by increasing the surface area. Such an increase in size would lead to a decreased surface area : volume ratio, thereby increasing water retention at least at some time points. However, there were no evident size increases in the selected females - certainly nothing approaching the difference in size between males and females, which is visible to the naked eye - and we consider that any potential microscopic changes in size would be unlikely to produce the significant differences in survival we observed in the selected lines.

Insight into the link between the effects on $\mathrm{CH}$ levels, water content and desiccation resistance can be found by inspecting the genetic mapping of desiccation-related phenotypes in desat1 transgenic flies. The selective knock-down of desat1 expression in the female fat body simultaneously induced a decreased desiccation resistance and a markedly increased water content. Knocking down desatl expression in all relevant tissues (including the fat body) induced very similar effects: the reduction of both desiccation resistance and the ratio of

342 desaturated : linear alkanes. However, selective targeting of desat1 expression in oenocytes 343 strongly decreased the ratio of desaturated : linear alkanes, but did not affect desiccation 344 resistance or water content. These data suggest that the relative increase of internal water 345 content, but not the decreased ratio of desaturated : linear alkanes, is involved in reduced 346 desiccation resistance. We also found evidence that a higher amount of internal water may not 
347 provide a decisive advantage in resisting desiccation: both desatl fat-body targeted genotypes 348 [RC-Gal4 x IR] and [6908-Gal4 x IR] - showed a lower LT50 but no slope change, suggesting 349 that the earlier mean age of death shown by these transgenic flies may have been due to faster water loss compared to flies with a relatively lower water content.

It is possible to interpret these complex data in terms of insect physiology. One of the main functions of the fat body is to store and release energy, whereas oenocytes regulate lipid metabolism (Ferveur 1997; Gutierrez et al. 2007). Energy is stored in adipocyte fat-body cells in the form of fatty acids, glycerol, triglycerides, and glycogen, which is stored in a bulky hydrated form (Arrese \& Soulages 2010). The amount of glycogen, which is normally lower than that of fat, can fluctuate depending on locomotor activity and environmental conditions (Lorenz \& Anand 2004). For example, if the insect is subject to freezing, low humidity or diapause, the fat body accumulates fat and eliminates water through the activity-dependent regulation of aquaporin water channel genes (Izumi et al. 2007; Liu et al. 2011; Sinclair \& Renault 2010). We propose that in our desatl fat body-targeted flies the capacity to store lipids was affected. This would explain why, under our desiccation challenge, fat body knock-down transgenic flies lost water more rapidly and died earlier than controls.

Further insight into the link between desiccation resistance and $\mathrm{CH}$ levels can be found from examining the other mutants studied here. $r k^{1 / 4}$ mutant flies showed a highly increased $\Sigma \mathrm{CH}$ but a control D:L ratio of desaturated : linear alkanes. These flies died earlier than controls, showing that a high $\mathrm{CH}$ level, even combined with a control-like D:L ratio, cannot compensate for the underlying genetic defect. We suspect that the defective sclerotization process observed in the rk mutant affects the permeability of the adult cuticle: this could enhance both $\mathrm{CH}$ trafficking and water loss (Flaven-Pouchon et al. 2016; Gibbs 1998; Moussian 2010). Conversely, $r k / \mathrm{CyO}$ females with a single $r k$ mutant allele and a control-like $\mathrm{CH}$ profile showed

371 a lower LT50 and a shallower death slope than controls (this was not due to the CyO marker 372 associated with the SM2 balancer). The reduction in the RK gene product may lead to a slowing 373 down of water loss by changing the ultrastructure of the cuticle or of the spiracles (Chown 2002; Moussian 2010). The clear sex differences in desiccation resistance may also be related to differences in CH levels:" mature females express longer chain CHs than mature males (Antony \& Jallon 1982; Gibbs et al. 1997). This may explain why immature flies carrying CHs with longer carbon chains and higher number of double-bonds survived longer than mature flies under 
378 dry humidity condition (Fig S1). However, no change in desiccation resistance was induced by

379 the replacement of a alkene (7,11HD) by a closely related isomer (5,9HD) in Z30 mature

380 females, which showed similar desiccation responses to Di2 females (Fig 10).

\section{Conclusion}

382 Our three-pronged approach to desiccation resistance and its underlying genetic and 383 phenotypic components - selection, $\mathrm{CH}$ analysis and measurements of water content - provides 384 insight into this fundamental aspect of the ecological physiology of insects. Our data suggest that 385 desiccation resistance is not a simple phenotype: increased and decreased resistance depended on 386 different hierarchies of physiological factors. There was no consistent relationship between 387 increased resistance and various measures of cuticular hydrocarbon composition. 388 Dessication resistance cannot be easily narrowed down to one or two variables, as seen by the 389 different cuticular hydrocarbon composition of the strains studied here. Surprisingly, even the 390 ability to retain a relatively high proportion of water was not related to desiccation resistance 391 transgenic flies with the highest proportion of water ([RC-Gal4 x IR] and [6908-Gal4 x IR])

392 showed the lowest levels of resistance. These two genotypes also showed reduced D : L (alkenes

393 : alkanes) ratios underlining that a decline in desiccation resistance may occur in flies combining 394 a high water content and low alkene levels. Flies in which these characters were dissociated 395 showed no major change in desiccation resistance. All flies that died rapidly showed a very 396 reduced LT50, but no change in their lethality slope: this suggests that early death equally 397 affected all individuals of a given genotype.

398 Our study has revealed an intricate and non-linear relationship between desiccation 399 resistance, $\mathrm{CH}$ profile and internal water content in D. melanogaster flies. These three 400 phenotypes, which might be expected to show a simple relationship, turn out to have complex 401 physiological and genetic links. While desiccation resistance and the proportion of desaturated 402 CHs were tightly linked with both measures rapidly increasing after selection and persisting long 403 after selection had been relaxed, a high water content negatively affected resistance, especially in 404 association with a low level of desaturated CHs. 


\section{References}

406

407

408

409

410

411

412

413

414

415

416

417

418

419

420

421

422

423

424

425

426

427

428

429

430

431

432

433

434

435

436

437

438

439

440

441

442

443

444

Addinsoft. 2012. XLSTAT 2012, Data analysis and statistics with Microsoft Excel. Paris, France.

Andersen SO. 2010. Insect cuticular sclerotization: a review. Insect Biochemistry and Molecular Biology 40:166-178.

Antony C, and Jallon JM. 1982. The Chemical Basis For Sex Recognition In DrosophilaMelanogaster. Journal of Insect Physiology 28:873-880.

Arrese EL, and Soulages JL. 2010. Insect fat body: energy, metabolism, and regulation. Annual Review of Entomology 55:207-225. doi: 10.1146/annurev-ento-112408-085356

Baker JD, and Truman JW. 2002. Mutations in the Drosophila glycoprotein hormone receptor, rickets, eliminate neuropeptide-induced tanning and selectively block a stereotyped behavioral program. Journal of Experimental Biology 205:2555-2565.

Balabanidou V, A. K, M. M, G.J. B, C. T, M.P. J, S.J. M, G. C, A. A, A. L, S. A, J. H, H. R, G.J. L, and J. V. 2016. Cytochrome P450 associated with insecticide resistance catalyzes cuticular hydrocarbon production in Anopheles gambiae. Proc Natl Acad Sci USA 113:9268-9273.

Bousquet F, Chauvel I, Flaven-Pouchon J, Farine J-P, and Ferveur J-F. 2016. Dietary rescue of altered metabolism gene reveals unexpected Drosophila mating cues. Journal of Lipid Research 57:1-11. doi:10.1194/jlr.M064683

Bousquet F, and Ferveur JF. 2012. desat1: A Swiss army knife for pheromonal communication and reproduction? Fly 6:102-107.

Bousquet F, Nojima T, Houot B, Chauvel I, Chaudy S, Dupas S, Yamamoto D, and Ferveur JF. 2012. Expression of a desaturase gene, desat1, in neural and nonneural tissues separately affects perception and emission of sex pheromones in Drosophila. Proceedings of the National Academy of Sciences of the United States of America 109:249-254. 10.1073/pnas.1109166108

Chertemps T, Duportets L, Labeur C, Ueda R, Takahashi K, Saigo K, and Wicker-Thomas C. 2007. A female- biased expressed elongase involved in long-chain hydrocarbon biosynthesis and courtship behavior in Drosophila melanogaster. Proc Natl Acad Sci 104:4273-4278.

Chown SL. 2002. Respiratory water loss in insects. Comp Biochem Physiol A Mol Integr Physiol 133:791-804.

da Silva SM, Lavander HD, de Santana Luna MM, de Melo Eloi da Silva A, Gálvez AO, and Coimbra MR. 2015. Artemia franciscana as a vector for infectious myonecrosis virus (IMNV) to Litopenaeus vannamei juvenile. Journal of Invertebrate Pathology 126:1-5.

Dallerac R, Labeur C, Jallon JM, Knippie DC, Roelofs WL, and Wicker-Thomas C. 2000. A Delta 9 desaturase gene with a different substrate specificity is responsible for the cuticular diene hydrocarbon polymorphism in Drosophila melanogaster. Proceedings of the National Academy of Sciences of the United States of America 97:9449-9454.

Demerec M. 1950. Biology of Drosophila. John Wiley: John Wiley. 
445 Dow JA. 2009. Insights into the Malpighian tubule from functional genomics. Journal of Experimental Biology 212:435-445.

447

448

449

450

451

452

453

454

455

456

457

458

459

460

461

462

463

464

465

466

467

468

469

470

471

472

473

474

475

476

477

478

479

480

481

482

483

484

Eigenbrode SD, and Espelie KE. 2003. Effects of plant epicuticular lpids on insect herbivores. Annual Review of Entomology 40:171-194.

Everaerts C, Farine JP, Cobb M, and Ferveur JF. 2010. Drosophila cuticular hydrocarbons revisited: mating status alters cuticular profiles. PLoS One 5:e9607. doi:10.1371/journal.pone.0009607

Fang S, Takahashi A, and Wu CI. 2002. A mutation in the promoter of desaturase 2 is correlated with sexual isolation between drosophila behavioral races. Genetics 162:781-784.

Ferveur JF. 1997. The pheromonal role of cuticular hydrocarbons in Drosophila melanogaster. BioEssays 19:353-358.

Ferveur JF. 2005. Cuticular hydrocarbons: Their evolution and roles in Drosophila pheromonal communication. Behavior Genetics 35:279-295.

Flaven-Pouchon J, Farine JP, Ewer J, and Ferveur JF. 2016. Regulation of cuticular hydrocarbon profile maturation by Drosophila tanning hormone, bursicon, and its interaction with desaturase activity. Insect Biochemistry and Molecular Biology 78:87-96.

Folk DG, and Bradley TJ. 2004. Evolved patterns and rates of water loss and ion regulation in laboratory-selected populations of Drosophila melanogaster. Journal of Experimental Biology 206:2779-2786.

Gibbs A, and Pomonis JG. 1995. Physical properties of insect cuticular hydrocarbons: the effects of chain length, methyl-branching and unsaturation. Comp Biochem Physiol B Biochem Mol Biol 112:243-249.

Gibbs AG. 1998. Water-proofing properties of cuticular lipids. American Zoologist 38:471-482.

Gibbs AG, Chippindale AK, and Rose MR. 1997. Physiological mechanisms of evolved desiccation resistance in Drosophila melanogaster. Journal of Experimental Biology 200:1821-1832.

Gibbs AG, and Rajpurohit S. 2010. Cuticular lipids and water balance. In: Blomquist GJ, and Bagnères A-G, eds. Insect Hydrocarbons: Biology, Biochemistry and Chemical Ecology. Cambridge, UK.: Cambridge University Press, 100-120.

Gołębiowski M, Maliński E, Boguś MI, Kumirska J, and Stepnowski P. 2008. The cuticular fatty acids of Calliphora vicina, Dendrolimus pini and Galleria mellonella larvae and their role in resistance to fungal infection. . Insect Biochemistry and Molecular Biology 38:619-627.

Grillet M, Everaerts C, Houot B, Ritchie MG, Cobb M, and Ferveur JF. 2012. Incipient speciation in Drosophila melanogaster involves chemical signals. Sci Rep 2:224. 10.1038/srep00224

Gutierrez E, Wiggins D, Fielding B, and Gould AP. 2007. Specialized hepatocyte-like cells regulate Drosophila lipid metabolism. Nature 445:275-280.

Hadley NF. 1994. Ventilatory patterns and respiratory transpiration in adult terrestrial insectss. Physiological Zoology 67:175-189. 
485

486

487

488

489

490

491

492

493

494

495

496

497

498

499

500

501

502

503

504

505

506

507

508

509

510

511

512

513

514

515

516

517

518

519

520

521

522

523

Harwood BN, Fortin J-P, Gao K, Chen C, Beinborn M, and Kopin AS. 2013. Membrane tethered bursicon constructs as heterodimeric modulators of the Drosophila G protein-coupled receptor Rickets. Molecular Pharmacology 83:814-821.

Honegger H-W, Dewey EM, and Ewer J. 2008. Bursicon, the tanning hormone of insects: recent advances following the discovery of its molecular identity. Journal of Comparative Physiology A 194:989-1005.

Houot B, Bousquet F, and Ferveur JF. 2010. The consequences of regulation of desat1 expression for pheromone emission and detection in Drosophila melanogaster. Genetics 185:1297-1309. 10.1534/genetics.110.117226

Houot B, Cazalé-Debat L, Fraichard S, Everaerts C, Saxena N, Sane SP, and Ferveur J-F. 2017. Gene regulation and species-specific evolution of free-flight odor-tracking in Drosophila. Molecular Biology and Evolution. doi: 10.1093/molbev/msx241

Howard RW, and Blomquist GJ. 2005. Ecological, behavioral, and biochemical aspects of insect hydrocarbons. Annual Review of Entomology 50:371-393.

Izumi Y, Sonoda S, and Tsumuki H. 2007. Effects of diapause and cold-acclimation on the avoidance of freezing injury in fat body tissue of the rice stem borer, Chilo suppressalis Walker. Journal of Insect Physiology 53:685-690.

Jallon JM. 1984. A Few Chemical Words Exchanged By Drosophila During Courtship And Mating. Behavior Genetics 14:441-478.

Liu K, Tsujimoto H, Cha S-J, Agre P, and Rasgon JL. 2011. Aquaporin water channel AgAQP1 in the malaria vector mosquito Anopheles gambiae during blood feeding and humidity adaptation. Proc Natl Acad Sci USA 108:6062-6066.

Locke M. 1966. The structure and formation of the cuticulin layer in the epicuticle of an insect, Calpodes ethlius (Lepidoptera, Hesperiidae). Journal of Morphology 118:461-494. 10.1002/jmor.1051180403

Lorenz MW, and Anand AN. 2004. Changes in the biochemical composition of fat body stores during adult development of female crickets, Gryllus bimaculatus. Archives of Insect Biochemistry and Physiology 56:110-119.

Moussian B. 2010. Recent advances in understanding mechanisms of insect cuticle differentiation. Insect Biochemistry and Molecular Biology 40:363-375.

Qiu Y, Tittiger C, Wicker-Thomas C, Le Goff G, Young S, Wajnberg E, Fricaux T, Taquet N, Blomquist GJ, and Feyereisen R. 2012. An insect-specific P450 oxidative decarbonylase for cuticular hydrocarbon biosynthesis. Proc. Natl. Acad. Sci. U. S. A. 109, . Proc Natl Acad Sci USA 109:14858-14863.

Robertson JL, and Preisler HK. 1992. Pesticide bioassays with Arthropods. Boca Raton, FL: CRC Press / Taylor \& Francis Group.

Savarit F, and Ferveur JF. 2002. Temperature affects the ontogeny of sexually dimorphic cuticular hydrocarbons in Drosophila melanogaster. Journal of Experimental Biology 205:3241-3249. 
524 Savarit F, Sureau G, Cobb M, and Ferveur JF. 1999. Genetic elimination of known pheromones

525

526

527

528

529

530

531

533

534

535

536

537

538 reveals the fundamental chemical bases of mating and isolation in Drosophila. Proceedings of the National Academy of Sciences of the United States of America 96:9015-9020.

Sinclair BJ, and Renault D. 2010. Intracellular ice formation in insects: unresolved after 50 years? Comp Biochem Physiol A Mol Integr Physiol 155:14-18. doi: 10.1016/j.cbpa.2009.10.026.

Toolson EC, and Hadley NF. 1979. Seasonal effects on cuticular permeability and epicuticular lipid composition in Centruroides sculpturatus (Scorpiones: Buthidae): correlation with thermal effects on cuticular permeability. Journal of Insect Physiology 25:271-275.

Tukey JW. 1977. Exploratory Data Analysis.

Wigglesworth VB. 1945. Transpiration through the cuticle of Insects. Journal of Experimental Biology 21:97-114.

Wigglesworth VB. 1988. The control of pattern as seen in the integument of an insect. BioEssays 9:23-27. 


\section{Figure 1}

Experimental selection of desiccation resistance line.

(A) Flies were kept in single sex groups of approximately 50 individuals in empty glass vials. 6 or 7 of these glass vials were packed inside an airtight transparent plastic box that was seeded with a layer of silicagel crystals to maintain a low relative humidity $(20 \pm 1 \%)$. The box was placed on a hot plate at $25 \pm 0.2^{\circ}$. Four boxes were simultaneously tested. Every two hours, the number of dead flies was counted, providing a measure of survival over time. This allowed us to estimate when $50 \%$ flies died (lethality time $50 \%=$ LT50), using logistic regression. The slope of the lethality curve was also determined to evaluate the relative lethality per hour. At the end of each experiment, the few surviving flies (maximum 1-2\% of all flies) were transferred into fresh food vials and mated with siblings to produce the next generation. (B) Arrows indicate the generations of the experiment. Plain arrows indicate the generations during which experimental selection for desiccation (light blue) and/or phenotypic measurements $(\mathrm{CH}=$ cuticular hydrocarbons; $\mathrm{W}=$ weight; $\mathrm{Fec}=$ fecundity) were carried out. Dashed arrows indicate the generations during which no measurement or selection was carried out. 
A

\section{Experimental and control genotypes}

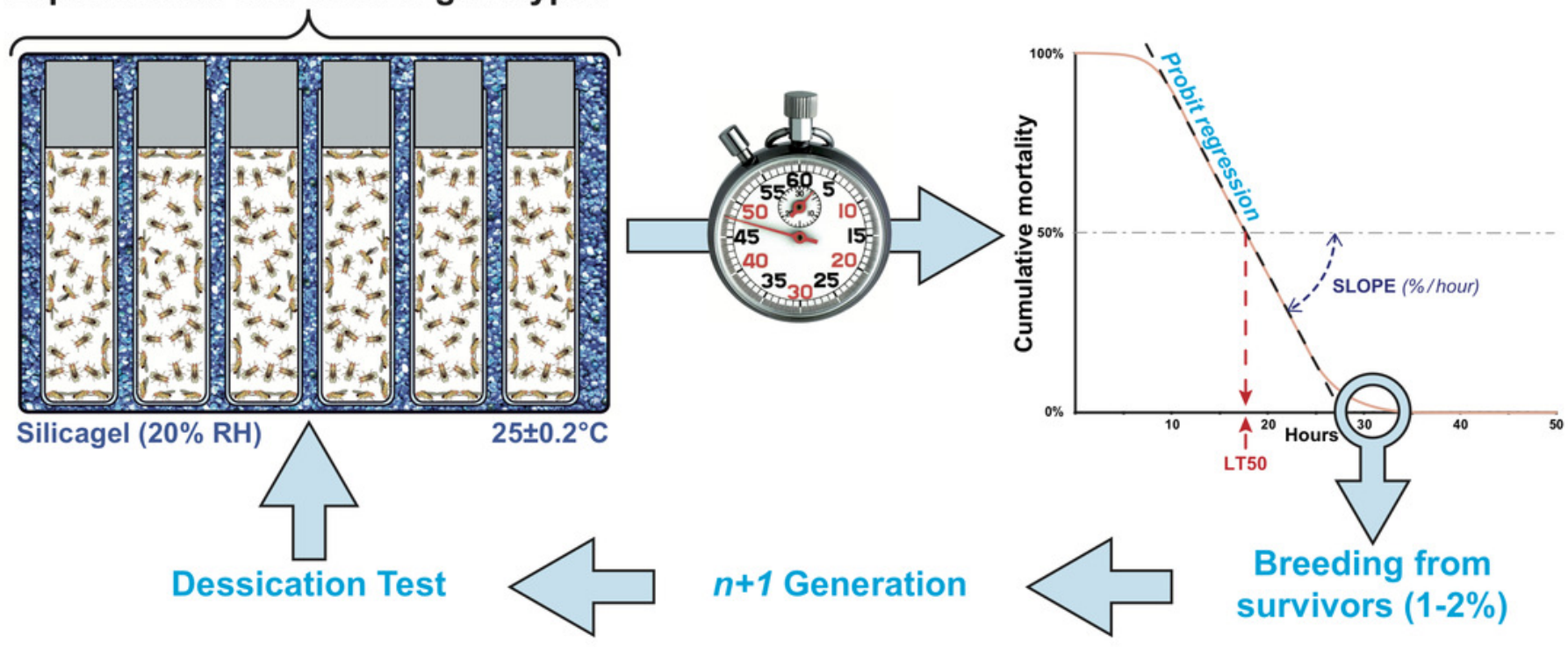

B

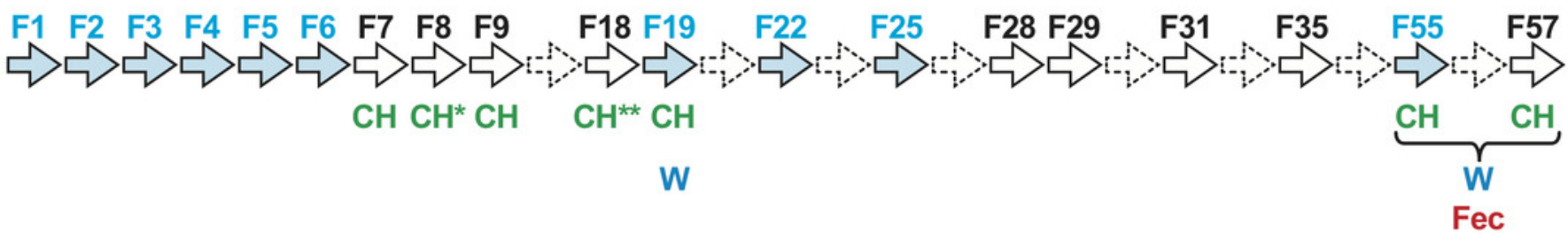




\section{Figure 2 (on next page)}

Survival in females selected for desiccation resistance over the first six generations.

Female flies were selected using the experimental procedure described in Fig. 1. (A) For each generation (F1 to F6), the curves represent the survival measured in various genotypes (dashed line $=$ Di2 control line, cyan line $=77 \mathrm{~S}$ selected lines pooled, magenta line $=$ backcross between $77 \mathrm{~S}$ females and unselected sibling males; at F1, three selected lines are shown). (B) At each generation, the two box-plots represent the LT50 and the lethality slope using colors similar to those of the corresponding genotypes. Data are shown as box plots representing the $50 \%$ median data (the small horizontal bar indicates the median value while the plain dot represents the mean). The whiskers shown below and above each box represent the first and third quartiles, respectively. Stars or different letters indicate significant differences. After excluding extreme outliers using Tukey's method, LT50 and slopes were tested using a Kruskall-Wallis test completed by a Conover-Iman multiple pairwise comparisons at level $p=0.05$ (with a Bonferroni correction) or with a Mann-Whitney test. ***: $p<0.001 ; * *: p<0.01 ; *: p<0.05$. The absence of a letter or stars indicates that no significant difference was detected. $N=5-17$ (except $5 \mathrm{~S}$ line at F1 and 77sxDi2 line at F3 where $N=3$ ). A similar selection procedure was carried out on males between the F1 and F6 generations (Fig. S2). 


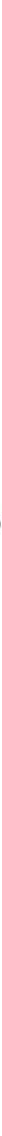




\section{Figure 3 (on next page)}

Survival in females of selected lines between F18 and F57.

Female flies were selected at the indicated generations using the procedure described in

Figs. $1 \& 2 . N=5-22$ (except Di2 line at F18: $N=4$, and at F25, $28 \& 29: N=3$ ). For parameters and statistics, see Fig. 2 legend. 


\section{Figure 4 (on next page)}

Principal cuticular hydrocarbons in flies of selected lines following relaxation of selection.

Cuticular hydrocarbon levels ( $\mathrm{CHs}$ ) were measured in F7, F8 and F9 females and in F8 males separately in the six $77 \mathrm{~S}$ lines $\left(77 \mathrm{~S}_{0}-77 \mathrm{~S}_{5}\right)$ experimentally selected for desiccation resistance (F1-F6; see Fig. 2). Here, we show the total absolute amount of $\mathrm{CHs}(\Sigma \mathrm{CH}$ in $\mu \mathrm{g}, \mathbf{A})$ and the ratio of Desaturated : Linear saturated CHs ( $\mathrm{D}: \mathrm{L}$ ratio; B) This ratio was calculated using the formula ([D - L] /[D + L]). $N=5-20$ for females and $N=9-14$ for males. For statistics, see Fig. 2 legend. We also determined the absolute (Q) and relative (\%) amounts of desaturated CHs (alkenes) and of linear saturated CHs (alkanes) (Fig. S3). 


\section{Figure $\mathbf{5}$ (on next page)}

Principal cuticular hydrocarbon levels in females of selected lines between F18 and F57.

$\Sigma \mathrm{CH}(\mathbf{A})$ and $\mathrm{D}: \mathrm{L}$ ratio (B) were measured in $\mathrm{F} 18, \mathrm{~F} 19, \mathrm{~F} 55$ and $\mathrm{F} 57$ females. As well as control unselected Di2 females, six $77 \mathrm{~S}$ lines $\left(77 \mathrm{~S}_{0}-77 \mathrm{~S}_{5}\right)$ were tested in F18 and F19; only 4 of these lines $\left(77 S_{1}-77 S_{4}\right)$ survived to F55 and F57. The 77S-Sel lines (77S-Sel1 - 77S-Sel4) that were tested at F57 were the offspring of F55 reselected females from their respective $77 \mathrm{~S}$ lines (e.g. $77 \mathrm{~S}_{1}$ females yielded the 77S-Sel1 line). $N=7-38$. For more information on parameters, lines and statistics, see legends of Fig.2 and Fig.4. The absolute (Q) and relative (\%) amounts of desaturated $\mathrm{CHs}$ (alkenes) and of linear saturated $\mathrm{CHs}$ (alkanes) determined in these flies are shown in Fig. $\mathrm{S4}$. 


\section{Figure 6 (on next page)}

Fresh : dry weight ratio in females of selected lines.

Groups of 10 freshly killed females were weighed (fresh weight) and after 24 hours desiccation were weighed again (dry weight). The fresh : dry weight ratio of each group was calculated. Females were weighed at F19 (A) and F57 (B). $N=6-20$. For more information on genotypes and statistics, see legends to Figs. $2,4 \& 5$. 

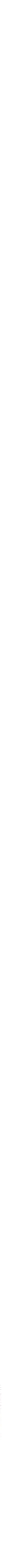

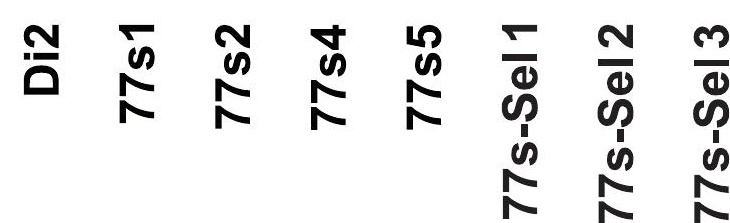




\section{Figure 7 (on next page)}

Desiccation resistance in various desat1 transgenic females.

To test the effect of desat1 knock-down expression in various desat1-expressing tissues, we used the female progeny of matings between transgenic females carrying the UAS-desat1-IR transgene (IR) and transgenic males either carrying each desat1 putative regulatory region fused with Gal4 (PRR-Gal4) corresponding to each desat1 transcript (RA, RC, RE, RB, RD, $\mathrm{RDiO})$, or the complete desat1 regulatory region $(6908 \mathrm{bp}=6908)$. Di2 control females and female progeny resulting of matings between IR females and Di2 males were also tested (Di2; Di2-IR; left box plots). The LT50 (A) and the lethality slope (B) of all these genotypes were determined. To control for the effect of each desat1 PRR-Gal4 transgene on the LT50 (C) and lethality slope (D), we used flies from matings between Di2 females and PRR-Gal4 males, alongside Di2-IR and Di2 control females. $N=5-13$. For more information on parameters and statistics, see Fig. 2 legend. 


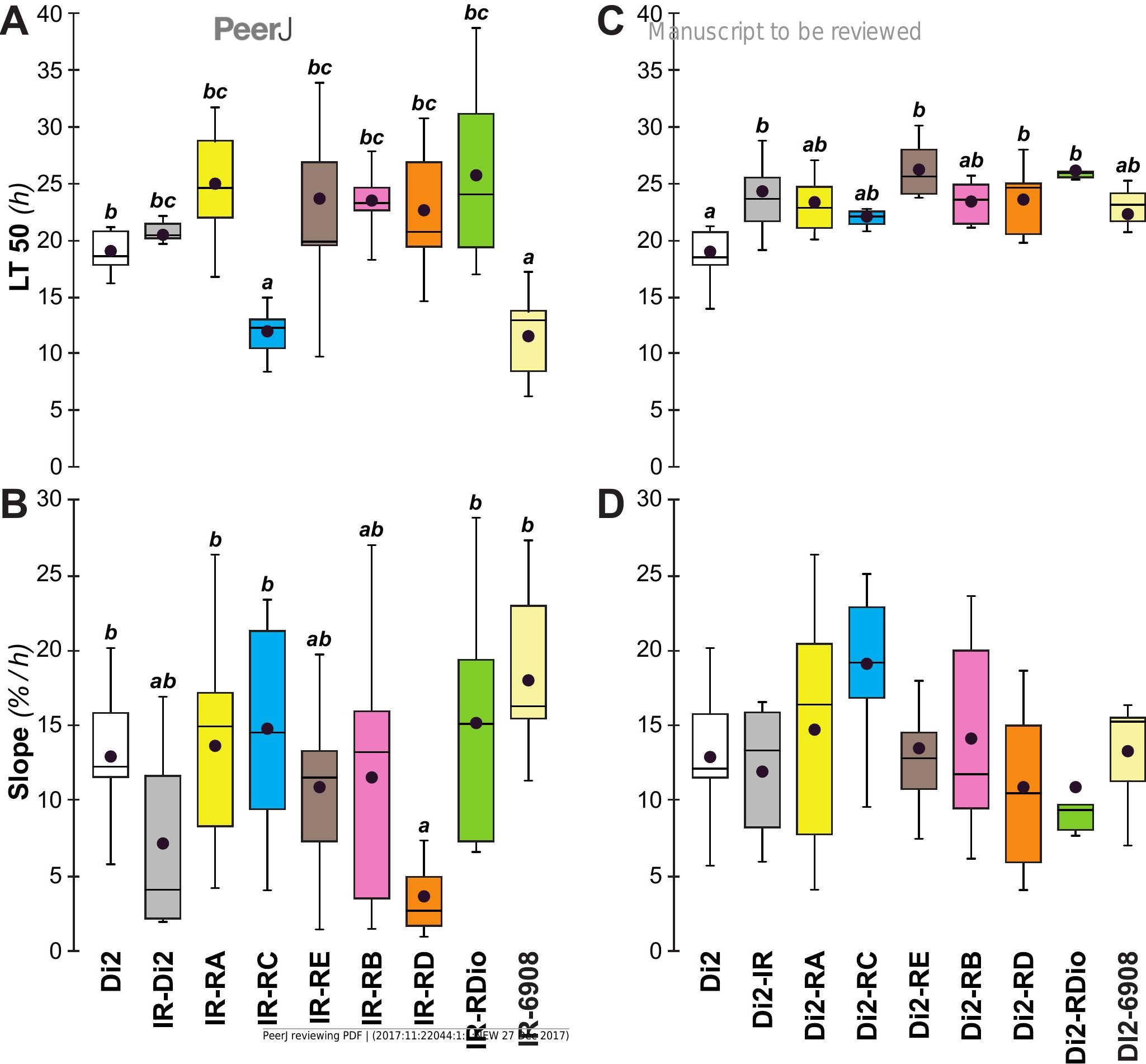




\section{Figure 8 (on next page)}

Principal cuticular hydrocarbons in various desat1 transgenic females.

$\mathrm{CH}$ levels were measured in all transgenic and control female flies tested for desiccation resistance (see Fig. 7). Transgenic females combining a maternal IR transgene with a paternal PRR-Gal4 or 6908 transgene were tested at both F55 (A) and F57 (B). Di2, Di2/w and Di2-IR control females were tested (left box-plots) either at F55 (A) or at F57 (B). Control genotypes carrying a paternal copy of each PRR-Gal4 transgene or of the 6908 transgene combined with a maternal Di2 genome were also tested $(\mathbf{C})$. Alongside these control genotypes, we also tested the effect of the IR transgene in the Di2 background (second boxplot from the left. $N=7-16$. For more information on $\mathrm{CHs}$, genotypes and statistics, see legends to Figs. $4 \& 7$. 


\section{Figure 9 (on next page)}

Fresh : dry weight ratio in various desat1 transgenic females.

$N=5-15$. For more information on genotypes and statistics, see legends to Figs. $2 \& 7$. 

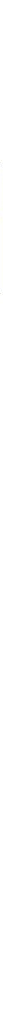

$\begin{array}{lll}B & \\ & & \\ 3.0 \\ 3.5\end{array}$

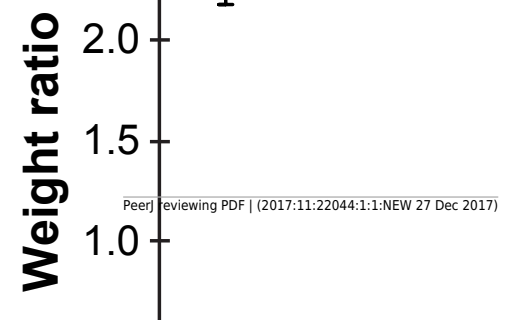

1.0
0.5
0.0

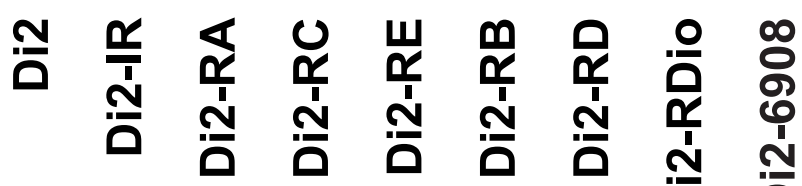
$\overline{0}$ 
Figure 10 (on next page)

Survival to desiccation in various cuticular hydrocarbon variant females.

The LT50 (A) and lethality slope (B) of various $\mathrm{CH}$ variants was measured. We also assayed the resistance of flies from these lines: control Di2, Di2/w (combining the Di2 genome with the $w^{1118}$ white-eye mutation), Zimbabwe 30 line $(\mathrm{Z30})$, ricket ${ }^{1 / 4}$ double trans-heterozygote mutant $\left(r k^{1} / r k^{4}\right)$, $r k$ heterozygotes carrying the SM2 balancer $\left(r k / \mathrm{CyO}=r k^{1} / \mathrm{CyO}\right.$ and $\left.r k^{4} / \mathrm{CyO}\right)$, and Di2/w flies carrying the SM2 balancer (CyO/w). $N=10-30$ (except for CyO/w line: $N=3$ ). For more information on statistics, see legend to Fig. 2. 
$\mathbf{A}^{35} \mathrm{~T}$

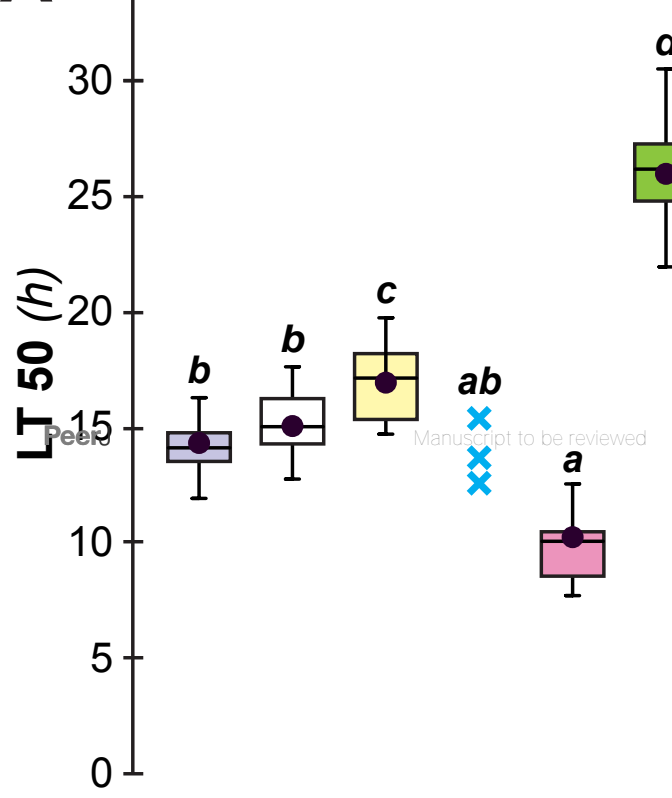

B $^{30} \mathrm{~T}$

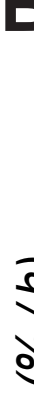

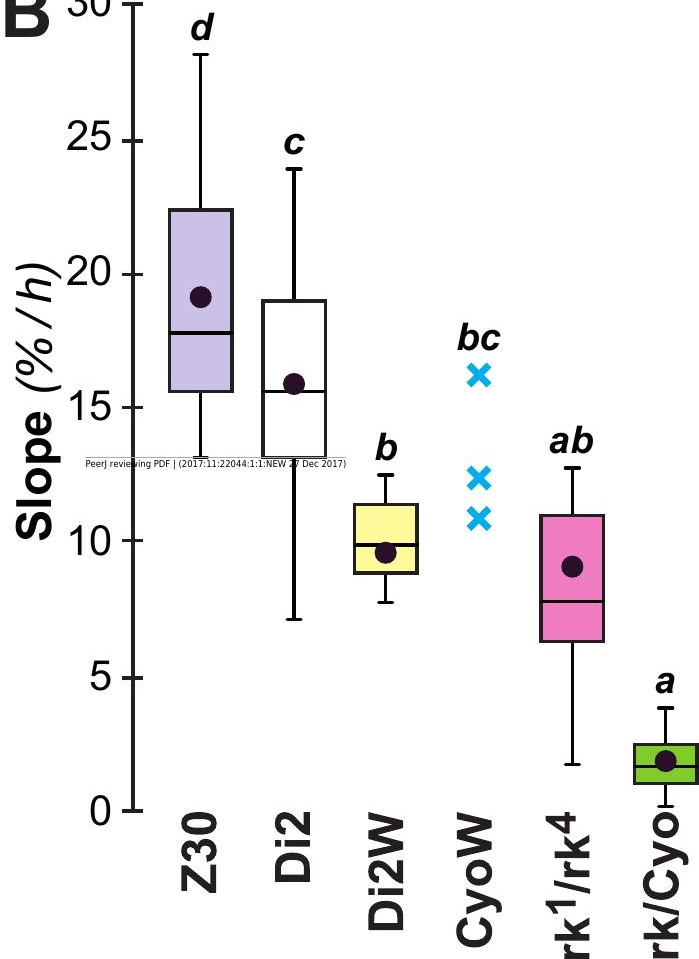

